\title{
Graphite Classification Based on Improved Convolution Neural Network
}

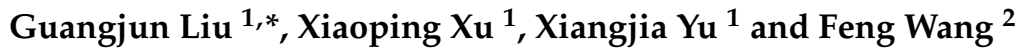 \\ 1 School of Sciences, Xi'an University of Technology, Xi'an 710054, China; xpxuxaut@sina.com (X.X.); \\ xjyuxaut@sina.com (X.Y.) \\ 2 School of Mathematics and Statistics, Xi'an Jiaotong University, Xi'an 710049, China; wangf@mail.xjtu.edu.cn \\ * Correspondence: liuguangjun202106@126.com
}

Citation: Liu, G.; Xu, X.; Yu, X.; Wang, F. Graphite Classification Based on Improved Convolution Neural Network. Processes 2021, 9 , 1995. https://doi.org/10.3390/ pr9111995

Academic Editor:

Roberta Campardelli

Received: 11 October 2021

Accepted: 4 November 2021

Published: 9 November 2021

Publisher's Note: MDPI stays neutral with regard to jurisdictional claims in published maps and institutional affiliations.

Copyright: (c) 2021 by the authors. Licensee MDPI, Basel, Switzerland. This article is an open access article distributed under the terms and conditions of the Creative Commons Attribution (CC BY) license (https:// creativecommons.org/licenses/by/ $4.0 /)$.

\begin{abstract}
In the development of high-tech industries, graphite has become increasingly more important. The world has gradually entered the graphite era from the silicon era. In order to make good use of high-quality graphite resources, a graphite classification and recognition algorithm based on an improved convolution neural network is proposed in this paper. Based on the self-built initial data set, the offline expansion and online enhancement of the data set can effectively expand the data set and reduce the risk of deep convolution neural network overfitting. Based on the visual geometry group 16 (VGG16), residual net 34 (ResNet34), and mobile net Vision 2 (MobileNet V2), a new output module is redesigned and loaded into the full connection layer. The improved migration network enhances the generalization ability and robustness of the model; moreover, combined with the focal loss function, the superparameters of the model are modified and trained on the basis of the graphite data set. The simulation results illustrate that the recognition accuracy of the proposed method is significantly improved, the convergence speed is accelerated, and the model is more stable, which proves the feasibility and effectiveness of the proposed method.
\end{abstract}

Keywords: graphite; classification; transfer learning; focal loss; convolution neural network

\section{Introduction}

At present, nonmetallic mineral resources are widely used in emerging materials, energy, national defense, aerospace industry, and other fields [1-7]. Its strategic significance is gradually improved and its application field is expanding. Therefore, the research on graphite is of great significance [8-14]. In general, graphite can be divided into two categories: natural graphite and artificial graphite. They are similar in structure and physicochemical properties, but their use paths are quite different. In metallurgy, machinery industry, chemical industry, and many other application fields, it is particularly important to identify the type of graphite. If the two are confused, decision-making errors will result, leading to much resource waste and economic losses. Therefore, from the perspective of strategic demand, research on the intelligent classification of graphite has great far-reaching significance for the classification management of graphite resources.

Most of the traditional methods to identify graphite are microscope identification or chemical identification. These methods have a large human factor and high time cost, which bring great challenges to the industry [15]. Currently, artificial intelligence research based on deep learning provides the most advanced solution for computer vision. As a deep learning method, convolution neural network (CNN) is popular in different disciplines of image recognition application. Small details that people do not notice can be easily distinguished by CNN. This algorithm can directly extract visual patterns from pixel images with minimal preprocessing. CNN structure introduced by LeCun net (LeNet) architecture and Alex Krizhevsky net (AlexNet) makes CNN popular. Since then, CNN's popularity has grown exponentially with a variety of designs and applications [16-20]. 
Due to the high similarity of the four types of graphite images shown in Figure 1, the recognition rate of shallow $\mathrm{CNN}$ (such as AlexNet) is low, and the model is relatively simple, which may limit its classification accuracy. Considering the recognition accuracy, recognition time, and model parameters, VGG16, ResNet34, and MobileNet V2, which have more complex network structure and deeper layers, are selected to improve classification accuracy. These networks have less hardware requirements and lower computational complexity, and can effectively extract deep features of images while shortening training time. Furthermore, these networks avoid the complex method of manual feature extraction and improve the recognition accuracy. In view of the characteristics of graphite images and the small data set, the data set is preprocessed to achieve the purpose of data enhancement. At the same time, preprocessing resets the loading module of the migration network, debugs the output parameters of each layer connection, improves the convergence speed of the network, and adds a dropout layer optimization model to effectively integrate the migration network and the model.

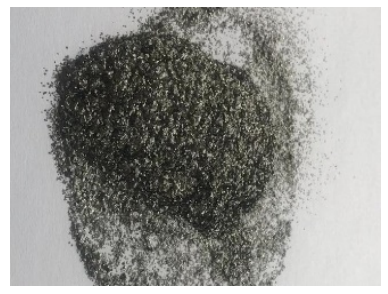

(a) Flake graphite

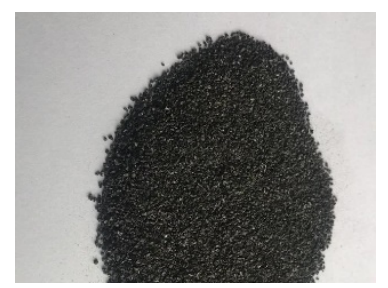

(c) Artificial graphite

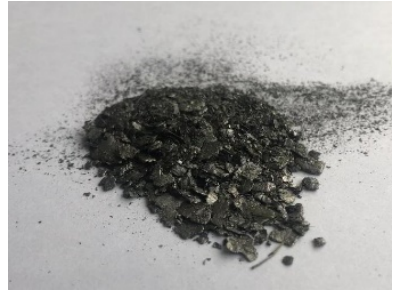

(b) Expanded graphite

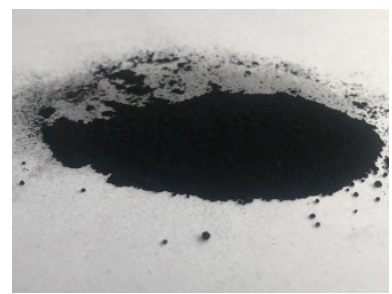

(d) Carbon black

Figure 1. Images showing four types of graphite.

Traditional CNN models mostly use cross entropy loss function (CEL), but CEL only considers the accuracy of correct tags, ignoring the difference of incorrect tags, so most researchers have studied the loss function. Triplet loss was proposed by Schroff in 2015 [21]. It is mainly used to train samples with small differences. However, the training process of this function is unstable, the convergence speed is slow, and it may fall into a local optimum. In 2017, for target detection of dense objects, heproposed focal loss function (FL), which not only solved the imbalance problem of samples, but also solved the imbalance problem of difficult and easy samples, making the network give more attention to difficult samples [22]. Recognizing that the graphite data set image difference is small, not easy to distinguish, and a difficult sample is far more challenging than an easy sample, this paper selects FL as the loss function to improve the stability of the network. The simulation results show that the improved migration network combined with FL has stronger robustness and generalization ability, higher recognition accuracy, and achieves an ideal recognition effect.

\section{Experimental Data}

\subsection{Data Set Construction}

In this paper, the experimental samples were collected in Neixiang County, Shaanxi Province, China, from the flake graphite, artificial graphite, expanded graphite, and carbon black samples of Ruixin high-temperature composite products. The samples were 
photographed from different directions for $3 \mathrm{~min}$ by using an iPhone rear camera at the same position, and the resolution of the video was $1080 \mathrm{p}$, the captured graphite video was extracted by frame, 1 frame per second. After screening, 715 sample images were obtained and classified. During the experiment, all the images were obtained from the saved data. The four types of graphite images are shown in Figure 1. However, it is difficult to obtain a variety of graphite data sets. The images collected sometimes have the characteristics of uneven distribution and few images, so it was necessary to enhance the data set. The data enhancement method is given in Section 2.2.

\subsection{Offline Expansion and Online Enhancement of Data Sets}

The data enhancement method can be divided into offline enhancement and online enhancement. Offline enhancement directly processes the data set, which is often used when the data set is small. Online enhancement is suitable for large data sets. After the batch data are obtained in the process of model training, the batch data are enhanced, such as rotation, translation, folding, and other corresponding changes. In this paper, when the initial data set was small, offline expansion was adopted to directly process the data set and increase the sample size of the data set, and the collected images were flipped vertically, rotated by 90 degrees, flipped around an axis, and underwent affine transformation; finally, 3575 images were obtained. Moreover, in order to reduce the overfitting problem of deep $\mathrm{CNN}$, online enhancement was used to process the data. Based on the characteristics of the graphite image, the training set images were randomly scrambled before input to reduce the influence of image order on the model. Each image in the training set was randomly cropped, the image pixels unified, and the image flipped horizontally according to the probability (here, setting $p=0.5$ ). Finally, the processed data set was normalized, and the data of each channel were regularized with the mean value 0.5 and standard deviation 0.5 , so as to achieve the purpose of data expansion and data enhancement.

According to the proportion, 2860 data sets (80\%) were randomly selected as the training set to train the network, and 715 data sets $(20 \%)$ were selected as the test set to verify the performance of the network. The training set and the test set were processed separately. The classification of data sets is shown in Table 1.

Table 1. Graphite data set.

\begin{tabular}{ccccc}
\hline & Flake Graphite & $\begin{array}{c}\text { Expanded } \\
\text { Graphite }\end{array}$ & $\begin{array}{c}\text { Artificial } \\
\text { Graphite }\end{array}$ & Carbon Black \\
\hline Data set & 1020 & 965 & 715 & 875 \\
Training set & 816 & 772 & 572 & 700 \\
Test set & 204 & 193 & 143 & 175 \\
\hline
\end{tabular}

\section{Experimental Principle and Method \\ 3.1. Transfer Learning}

In recent years, successful case studies regard deep CNN as the mainstream method to solve challenging computer vision tasks [23]. However, training a network from scratch requires many training data, much time, and a large graphic processing unit (GPU). We only have 3575 training and test images, and a small data set cannot train a deep CNN. Therefore, this paper combines the idea of transfer learning to train the convolution neural network.

Transfer learning refers to the process of using models that have been trained for different tasks, hoping that the model can have sufficient generalized information to solve new specific tasks [24]. This method uses CNN, a pretraining model on a huge database, to help learning target tasks [25]. The classified image in this paper is graphite, which has different visual performance, which has a visual performance different from cats and dogs. The visual performance learned from a large image may not be able to represent the graphite image very well, so it is necessary to modify the pretrained CNN structure to 
adapt to our task. Zeiler and Fergus provide evidence that the general image representation learned from the pretrained CNN is superior to the most advanced handmade features [26].

\subsection{Convolution Neural Network Model}

At present, common CNN include AlexNet, visual geometry group (VGG), residual net (ResNet), mobile net (MobileNet), etc.; the ready-made image representation learned from these deep networks is powerful and universal, and has been used to solve many visual recognition problems [27]. Considering the good performance of these ready-made $\mathrm{CNN}$ features, they have become the mainstream image features to solve most computer vision problems [28].

Deep convolution neural network has the characteristics of deep depth and good recognition effect. VGG16, ResNet34, and MobileNet V2 are representative CNNs in recent years, which have achieved excellent results in the field of image classification. VGG uses several smaller convolution kernels instead of larger ones to ensure that the network depth can continue to increase under the condition of the same receptive field. VGG16 has 16 hidden layers (13 convolution layers and 3 fully connected layers), which is a classic CNN model for image classification in the deep learning framework. ResNet puts forward residual structure and uses convolution to reduce dimension, which makes ResNet have advantages in parameters, depth, width, and calculation cost without introducing additional parameters and increasing network calculation complexity. There are less than 4000 data sets in this paper, and the depth of ResNet34 model can be used effectively to train the samples. As a lightweight network proposed in 2018, MobileNet V2 always uses depthwise (DW) convolution to extract features, and proposes inverted residual, which can reduce the use of memory in implementation [29]. Based on these advantages, this paper establishes graphite classification and recognition model based on VGG16, ResNet34, and MobileNet V2 networks; the comparative analysis is shown in Table 2.

Table 2. Comparative analysis of models.

\begin{tabular}{cccc}
\hline Network & VGG16 & ResNet34 & MobileNet V2 \\
\hline Year & 2014 & 2015 & 2018 \\
Top-1 accuracy & $71.5 \%$ & - & $71.7 \%$ \\
Number of parameters & $138,357,544$ & $63,470,656$ & $4,253,864$ \\
Number of layers & 16 & 34 & - \\
BN & No & Yes & Yes \\
Residual structure & No & Yes & Yes \\
\hline
\end{tabular}

\subsection{Focus Loss Function}

In image classification, the loss function is generally cross entropy loss function. Cross entropy is the distance between the real output (probability) and the predicted output (probability); therefore, the smaller the value of cross entropy, the closer the two probability distribution. For the multiclassification of graphite, there are a large number of simple negative samples in the data set. The losses caused by these samples in the iterative process occupy the majority of the total losses, which may lead us to deviate from the correct optimization direction. To improve this situation, the focus loss function is used in this paper; the formula is shown in Equation (1).

$$
L_{\mathrm{FL}}=\left\{\begin{array}{l}
-\left(1-y^{\prime}\right)^{\gamma} \log y^{\prime}, y=1 \\
-y^{\gamma} \log \left(1-y^{\prime}\right), y=0
\end{array}\right.
$$

Equation (1) gives the contribution of classified samples to the loss. Due to the effect of modulation factor $\gamma$, the model weakens the error contribution of easy-to-identify samples, so that the model can focus on the hard-to-classify samples more effectively in training. After many experiments, $\gamma=0.25$ is set. On this basis, FL also introduces a balance factor $\alpha$, as shown in Equation (2). This factor can be used to balance the imbalance 
between the number of positive and negative samples, that is, class imbalance. Because the class imbalance problem of the self-built graphite data set is not very prominent, we set $\gamma=$ NAN.

$$
L_{F L}=\left\{\begin{array}{l}
-\alpha\left(1-y^{\prime}\right)^{\gamma} \log y^{\prime}, y=1 \\
-(1-\alpha) y^{\gamma} \log \left(1-y^{\prime}\right), y=0
\end{array}\right.
$$

\subsection{Improved Migration Network Model}

Combined with transfer learning, the model training process designed in this paper includes the following three stages: data expansion, transfer learning, and superparameter adjustment. The model training process is shown in Figure 2.

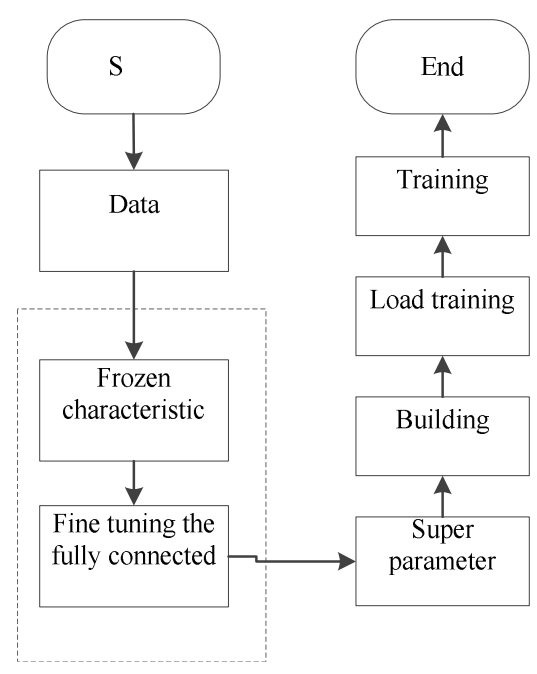

Figure 2. Flow chart of algorithm implementation.

Before loading new output modules, a global average pooling layer is added. Next, the SoftMax nonlinear final classification layer is used to set the final output to 4 . Finally, the Adam optimizer is used to fine tune 100 epochs of all models. The initial learning rate is 0.0002 , Nesterov momentum is 0.9 , and batch size is 32 .

In the above algorithm flow, this paper improves the three networks used, freezes the feature layer of each model architecture, redesigns new output modules and loads them into the full connection layer of their respective networks. The new full connection layer is shown in Figure 3.

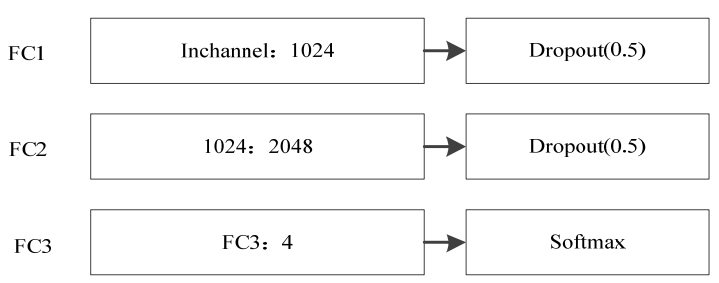

Figure 3. New output module.

In the new output module, three connection layers are set up to debug the output parameters of connection layers full connection 1 (FC1) and full connection 2 (FC2) to optimize the stability and generalization ability of the model. Because there are a large number of parameters in the full connection layer of the network, dropout is added in connection layers FC1 and FC2 to set the random loss probability $p=0.5$. This technology can reduce the complex co-adaptation of neurons and make the model more effective for fusion. In this way, the structure of the migration network is improved, as shown in Figure 4 . 


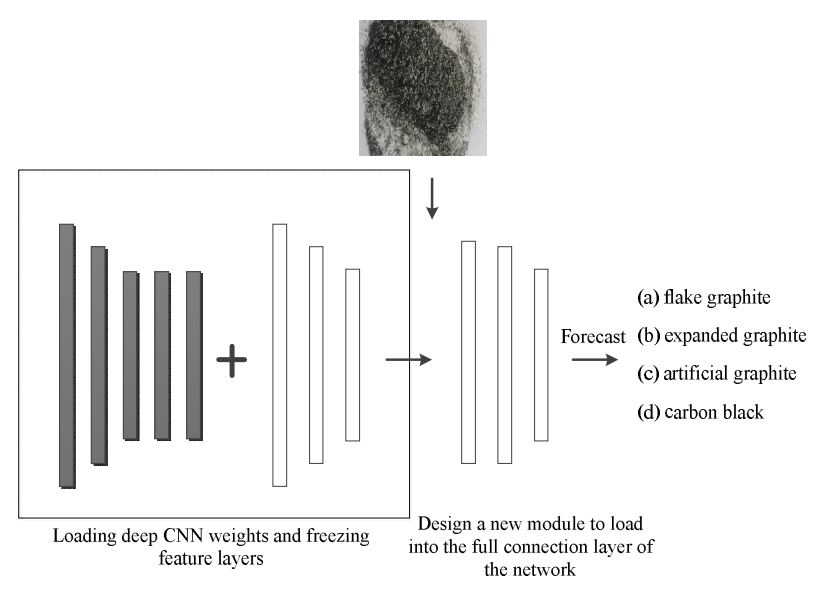

Figure 4. The structure of the improved migration network.

Herein, this model is called the improved convolutional neural network model based on transfer learning, which is applied to the graphite classification problem. The modified and optimized networks are renamed the improved visual geometry group 16 (I-VGG16), improved residual net 34 (I-ResNet34), and improved mobile net Vision 2 (I-MobileNet V2). On the basis of these improvements, the networks combined with FL loss function are recorded as I-VGG16 + FL, I-ResNet34 + FL, and I-MobileNet V2 + FL.

\section{Result Analysis}

\subsection{Evaluation Index and Environmental Configuration}

In this paper, the training effect of a network is evaluated by three indexes: accuracy $(a c c)$, loss, and running time. Test acc refers to the ratio of the model's output of correct results on the test set, and its definition formula is shown in Equation (3).

$$
a c c=\frac{n_{\text {correct }}}{n}
$$

where $n_{\text {correct }}$ denotes the number of correct network identifications in the test set and $n$ denotes the number of samples in the test set.

The training process of transfer learning is to minimize the loss function, and loss is the value of the loss function. In fact, the loss function calculates the mean square error (MSE, E) of the model on the test set.

$$
E=\frac{1}{n} \sum_{i}\left(\hat{y}-y^{\text {test }}\right)_{i}^{2}
$$

In this paper, accordingly, the epoch time is specified by the end of the whole network model. An epoch is a process in which all training samples are propagated forward and backward in the neural network model; that is, all training samples are trained once.

We used the 1.8.0 version of Python framework to complete the experimental simulation under the compiling environment of Python 3.8, and realized the image data preprocessing through transform.

\subsection{Test Results}

In order to verify the feasibility and effectiveness of the method presented, the graphite data set after data enhancement was imported into the method proposed in this paper. Through the experiment on the test set, the (i) unmodified model, (ii) improved model, and (ii) network model with the modified loss function, are all compared. Finally, the comparison curves of model loss and accuracy are obtained, as shown in Figures 5-7. 


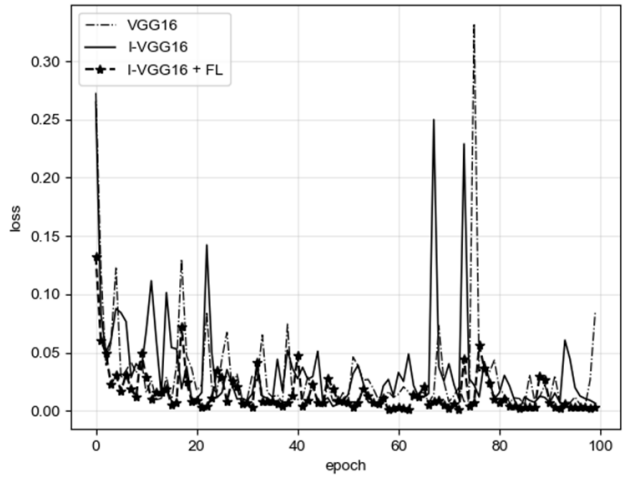

(a) Loss

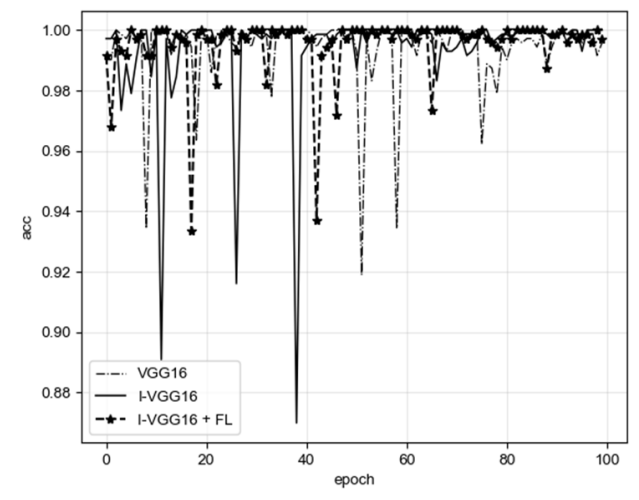

(b) Accuracy

Figure 5. VGG16 model loss and accuracy comparison chart.

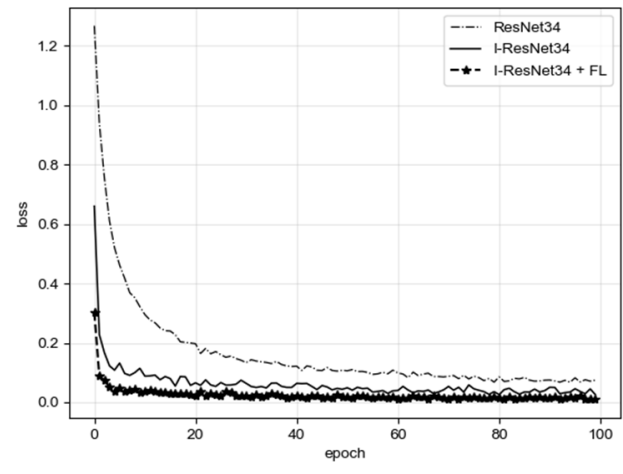

(a) Loss

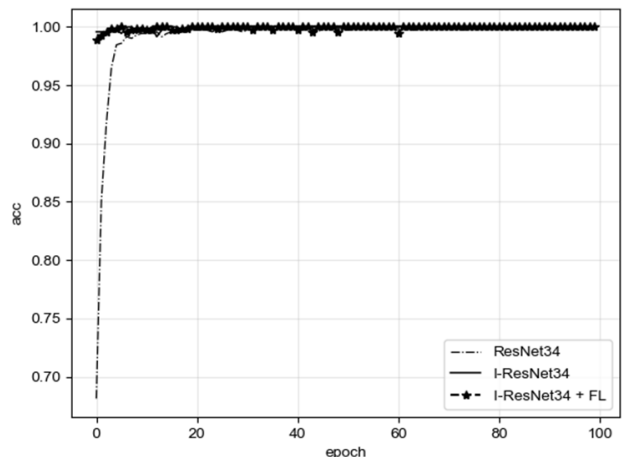

(b) Accuracy

Figure 6. ResNet34 model loss and accuracy comparison chart.

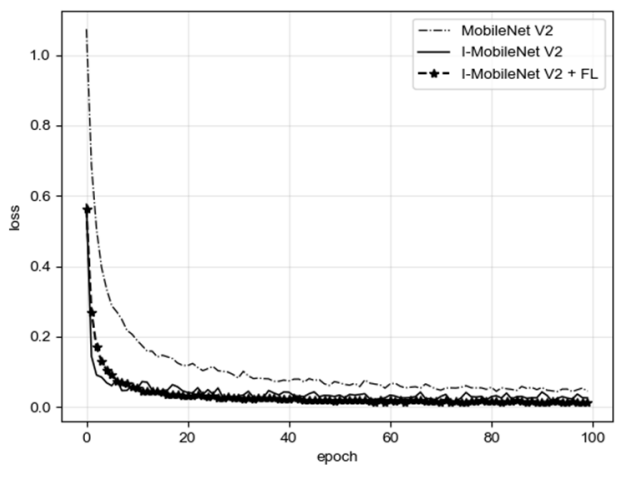

(a) Loss

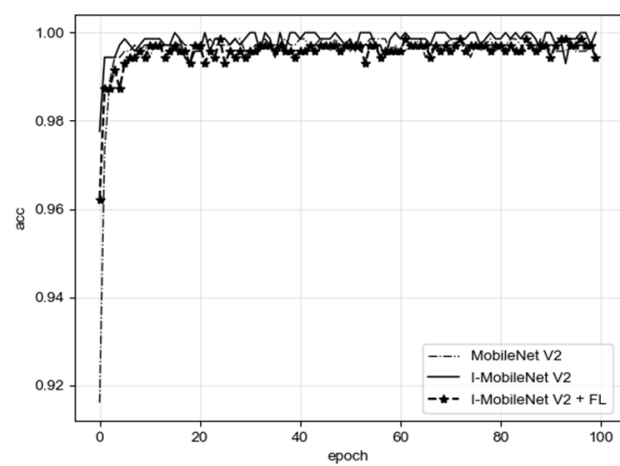

(b) Accuracy

Figure 7. MobileNet V2 model loss and accuracy comparison chart.

As shown in Figure 5, VGG16 has large loss value vibration, and the stability of the model is poor. After improvement of the model, the network performance of I-VGG16 and I-VGG16 + FL is obviously more stable and superior. Compared with VGG16, ResNet34 and MobileNet V2 have a deeper network and more stable model. It can be seen that the improved network effectively alleviates the problem of poor feature extraction ability caused by the secondary application of transfer learning. After using FL, the model is further optimized, the loss value is gradually reduced, and the accuracy is effectively improved, as shown in Figures 6 and 7. 
In order to better understand the improved CNN model, the loss value, accuracy, and training time of the final experimental results of nine models in this paper are shown in Table 3. Table 3 shows that although the accuracy and training time of VGG16 are relatively poor, the loss is relatively minimal; overall, the training time of ResNet34 is relatively short and the accuracy of the optimized model is also improved. MobileNet V2, as a lightweight network, can reach more than $99 \%$ recognition accuracy, and with the improvement of the loss function, the loss value gradually decreases, which proves that the combination of FL and the improved model can effectively focus on the difficult sample image of the graphite data set. This shows the feasibility and effectiveness of a convolutional neural network in graphite classification. The comparison chart of the loss value and accuracy of the improved CNN model of FL is shown in Figure 8, which intuitively shows that the improved model is more stable, can converge quickly in several epochs, and the accuracy is floating within $93 \%$, which proves the effectiveness of the improved algorithm.

Table 3. Comparison of loss value, accuracy, and training time of different algorithm models.

\begin{tabular}{cccc}
\hline Model & Loss Value $\left(\times \mathbf{1 0}^{-\mathbf{2}}\right)$ & Accuracy (\%) & Training Time (min) \\
\hline VGG16 & 0.36 & 92.93 & 74.73 \\
I-VGG16 & 0.16 & 93.71 & 76.13 \\
I-VGG16 + FL & 0.10 & 95.69 & 75.88 \\
ResNet34 & 6.75 & 93.29 & 40.13 \\
I-ResNet34 & 2.61 & 99.18 & 40.72 \\
I-ResNet34 + FL & 0.97 & 99.84 & 41.27 \\
MobileNet V2 & 4.37 & 98.57 & 39.10 \\
I-MobileNet V2 & 1.68 & 99.81 & 45.18 \\
I-MobileNet V2 + FL & 1.23 & 99.57 & 46.82 \\
\hline
\end{tabular}

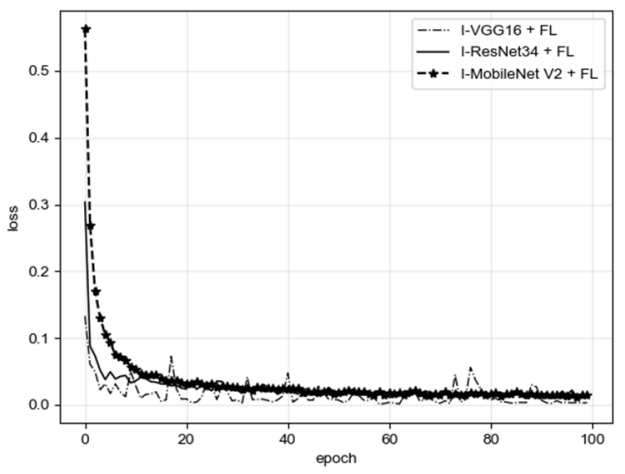

(a) Loss

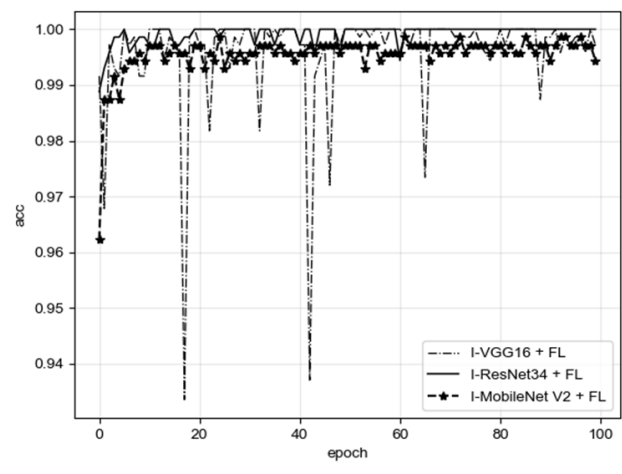

(b) Accuracy

Figure 8. Comparison of the improved CNN model combined with FL.

As can be seen from these experimental results, a graphite classification and recognition method using an improved convolution neural network is effective and achieves the purpose of the paper.

\section{Conclusions}

In this paper, an improved CNN combined with focal loss is applied to graphite image classification and recognition. The recognition model based on CNN does not need complicated image preprocessing steps in the early stage, and the data enhancement can also be completed online in the training process, which makes the model building process more concise. The graphite data set is expanded and enhanced to solve the problem of insufficient training samples. Through the simulation experiment on the processed data set, it is concluded that the redesigned full connection layer is helpful to improve the classification performance of the network. After optimizing the loss function, the 
performance of the network model is further improved by debugging the superparameters of the model, and the relatively high accuracy and low loss value of graphite classification are realized. In the future work, we will try deep networks other than the three kinds of CNN that were implemented. Therefore, it is our key research content in the future to explore the influence of different freezing ratios and freezing layers on the classification effect of the graphite data set.

Author Contributions: Conceptualization, X.X. and X.Y.; methodology, F.W.; software, X.X.; validation, X.X., X.Y. and F.W.; formal analysis, G.L.; investigation, G.L.; resources, X.X.; data curation, G.L.; writing—original draft preparation, X.X.; writing—review and editing, X.Y.; visualization, G.L.; supervision, X.X.; project administration, G.L.; funding acquisition, X.X. All authors have read and agreed to the published version of the manuscript.

Funding: This work is supported by the Innovation Capability Support Program of Shaanxi Province of China (Grants No. 2020PT-023), National Natural Science Foundation of China (Grants No. 11801438), and Natural Science Basic Research Plan in Shaanxi Province of China (Grants No. 2018JQ1089).

Institutional Review Board Statement: Not applicable.

Informed Consent Statement: Not applicable.

Data Availability Statement: The data sets used and/or analyzed during the current study are available from the corresponding author on reasonable request.

Conflicts of Interest: It is declared by the authors that this article is free of conflict of interest.

\section{References}

1. Wang, C.Y.; Zhao, J.L.; Du, X.H. Hydrogen production from ammonia borane hydrolysis catalyzed by non-noble metal-based materials: A review. J. Mater. Sci. 2021, 56, 2856-2878. [CrossRef]

2. Li, H.R.; Zhang, X.L.; Qi, Y.; Sun, R. Study on process of high pressure water jet cleaning mold of matrix PDC bit. Coal Mine Mach. 2021, 42, 93-96.

3. Ma, Y.J.; Fang, M.; Li, G.X.; Huang, M.; Zhang, N. Study on wear resistance and thermal stability of graphite reinforced TPU materials. Mod. Plast. Process. Appl. 2021, 33, 1-3.

4. Lu, J.J.; Liu, J.H.; Qian, G.Y.; Wang, Z.; Ma, J. Preparation of anode material for lithium-ion battery from waste silicon powder by ball milling. Min. Metall. 2021, 30,12-18.

5. Wu, E.H.; Li, J.; Hou, J.; Xu, Z.; Huang, P.; Jiang, Y.; Chen, F. Investigation on preparation and electrical properties of graphite $/ \mathrm{VO}_{2.4-x}$ composite powders. Rare Met. Cem. Carbides 2021, 49, 57-61.

6. $\quad$ Feng, L.L.; Chen, Y.; Li, J.G.; Tang, S.Y.; Du, J.Z.; Li, T.Y.; Li, X.G. Research progress in carbon-based composition molded bipolar plates. Chin. J. Eng. 2021, 43, 585-593.

7. Dong, Y.R.; Kong, G.L.; Zhang, Y.; Ma, L.; Wei, L.M. Preparation and application of micro silicon-graphite-carbon anodes for lithium-ion batteries. Micronanoelectron, Technol. 2021, 58, 379-385.

8. Liu, M.Q.; Jiao, Y.Y.; Qin, J.C.; Zhongjun, L.; Wang, J. Boron doped $\mathrm{C}_{3} \mathrm{~N}_{4}$ nanodots/nonmetal element (S, P, F, Br) doped C $3 \mathrm{~N}_{4}$ nanosheets heterojunction with synergistic effect to boost the photocatalytic hydrogen production performance. Appl. Surf. Sci. 2021, 541, 148558. [CrossRef]

9. Yang, S.Z.; Liang, J.; Feng, B.; Liu, P.; Yang, X.F.; Liu, Q.C. Study on carbon coating modification of nana-silicon/graphite anode materials. Funct. Mater. 2021, 52, 03130-03134.

10. Zhou, S.Z.; Nie, T.T.; Zhang, J.Y. Research and analysis of purification technology for graphite. Carbon Tech. 2021, 40, 60-62.

11. Cui, A.L.; Feng, G.X.; Zhao, Y.F. Synthesis and separation of mellitic acid and graphite oxide colloid through electrochemical oxidation of graphite in deionized water. Electrochem. Commun. 2009, 11, 409-412. [CrossRef]

12. Sheng, Z.H.; Lin, S.; Chen, J.J. Catalyst-free synthesis of nitrogen-doped graphene via thermal annealing graphite oxide with melamine and its excellent electrocatalysis. ACS Nano 2011, 5, 4350-4357. [CrossRef] [PubMed]

13. Laffont, L.; Pugliara, A.; Hungria, T.; Lacaze, J. Stem observation of a multiphase nucleus of spheroidal graphite. J. Mater. Res. Technol. 2020, 9, 4665-4671. [CrossRef]

14. Kumar, V.S.P.; Deshpande, P.A. Synergistic effect of metal-nonmetal substitution on oxygen activation in Pd/C- and Pd/Nsubstituted TiO2. Comput. Mater. Sci. 2019, 162, 349-358. [CrossRef]

15. Li, Y.; Zhang, S.Y. Present situation and prospect of high purity graphite production process. Sci. Technol. Innov. 2018, 22, 166-167.

16. Lecun, Y.; Bottou, L. Gradient-based learning applied to document recognition. Proc. IEEE 1998, 86, 2278-2324. [CrossRef]

17. Kim, S.; Song, W.J.; Kim, S.H. Double weight-based SAR and infrared sensor fusion for automatic ground target recognition with deep learning. Remote Sens. 2018, 10, 72. [CrossRef] 
18. Bouti, A.; Mahraz, M.A.; Riffi, J. A robust system for road sign detection and classification using LeNet architecture based on convolutional neural network. Soft Comput. 2020, 24, 121-128. [CrossRef]

19. Krizhevsky, A.; Sutskever, I.; Hinton, G.E. ImageNet classification with deep convolution Neural Networks. Adv. Neural Inf. Process. Syst. 2012, 25, 1097-1105.

20. Han, X.; Zhong, Y.; Cao, L. Pre-trained AlexNet architecture with pyramid pooling and supervision for high spatial resolution remote sensing image scene classification. Remote Sens. 2017, 9, 848. [CrossRef]

21. Schroff, F.; Kalenichenko, D.; Philbin, J. Facenet: A unified embedding for face recognition and clustering. In Proceedings of the IEEE Conference on Computer Vision and Pattern Recognition, Boston, MA, USA, 7-12 June 2015; pp. 815-823.

22. He, K.M.; Zhang, X.Y.; Ren, S.Q.; Jian, S. Deep residual learning for image recognition [EB/OL]. arXiv 2021, arXiv:1512.03385.

23. Simonyan, K.; Zisserman, A. Very deep convolutional networks for large-scale image recognition [EB/OL]. arXiv 2021, arXiv:1409.1556.

24. Lu, J.; Behbood, V.; Hao, P.; Zuo, H.; Xue, S.; Zhang, G. Transfer learning using computational intelligence: A survey. KnowledgeBased Syst. 2015, 80, 14-23. [CrossRef]

25. Tajbakhsh, N.; Shin, J.Y.; Gurudu, S.R.; Todd Hurst, R.; Kendall, C.B.; Gotway, M.B.; Liang, J. Convolutional neural networks for medical image analysis: Full training or fine tuning? IEEE Trans. Med. Imaging 2016, 35, 1299-1312. [CrossRef]

26. Zeiler, M.D.; Fergus, R. Visualizing and understanding convolutional net-works [EB/OL]. arXiv 2021, arXiv:1311.2901.

27. Razavian, A.S.; Azizpour, H.; Sullivan, J.; Carlsson, S. CNN features off-the-shelf: An astounding baseline for recognition. In Proceedings of the 2014 IEEE Conference on Computer Vision and Pattern Recognition Workshops, Columbus, OH, USA, 23-28 June 2014; pp. 512-519.

28. Azizpour, H.; Razavian, A.S.; Sullivan, J.; Maki, A.; Carlsson, S. From generic to specific deep representations for visual recognition [EB/OL]. arXiv 2021, arXiv:1406.5774.

29. Sandler, M.; Howard, A.; Zhu, M.L.; Zhmoginov, A.; Chen, L.-C. MobileNetV2: Inverted residuals and linear bottlenecks [EB/OL]. arXiv 2021, arXiv:1801.04381. 